Review Article

\title{
Acupuncture for Obstructive Sleep Apnea (OSA) in Adults: A Systematic Review and Meta-Analysis
}

\author{
Liaoyao Wang, ${ }^{1}$ Jia Xu, $^{2}$ Yijun Zhan, ${ }^{1}$ and Jian Pei $\mathbb{D}^{1}$ \\ ${ }^{1}$ Department of Acupuncture, Longhua Hospital, Shanghai University of Traditional Chinese Medicine, Shanghai 200032, China \\ ${ }^{2}$ Department of Acupuncture, Special Wards, Longhua Hospital, Shanghai University of Traditional Chinese Medicine, \\ Shanghai 200032, China
}

Correspondence should be addressed to Jian Pei; longhuaacup@aliyun.com

Received 22 October 2019; Accepted 26 December 2019; Published 6 March 2020

Academic Editor: Francesco Onida

Copyright (c) 2020 Liaoyao Wang et al. This is an open access article distributed under the Creative Commons Attribution License, which permits unrestricted use, distribution, and reproduction in any medium, provided the original work is properly cited.

Objective. Our aim was to assess the efficacy and safety of acupuncture for OSA patients with various severities of the disorder. Methods. Eight databases including PubMed, Cochrane Library, EMBASE, Web of Science, China National Knowledge Infrastructure (CNKI), Chongqing VIP (CQVIP), Wanfang Data, and Chinese Biomedical Literature Database (CBM) were comprehensively searched till July 2019. Randomized controlled trials (RCTs) testing acupuncture in the treatment of OSA were eligible for inclusion. Studies were selected for inclusion, and data were extracted by two authors independently. The Cochrane Collaboration's Risk of Bias Assessment Tool and RevMan software (version 5.3) were used to evaluate the quality of studies and conduct statistical analysis. Results. Nine RCTs with 584 participants were included. The trials covered acupuncture and electropuncture. Acupuncture caused clinically significant reductions in AHI (MD: -6.18; 95\% CI: -9.58 to $-2.78 ; Z=3.56, P=0.0004$ ) as well as in ESS (MD: $-2.84 ; 95 \%$ CI: -4.80 to $-0.16, Z=2.09, P=0.04$ ). AHI was reduced more in the subgroup analysis of moderate OSA patients (MD: $-9.44 ; 95 \%$ CI: -12.44 to $-6.45 ; Z=6.18, P<0.00001$ ) and severe OSA patients (MD: $-10.09 ; 95 \% \mathrm{CI}:-12.47$ to $-7.71 ; Z=8.31, P<0.00001$ ). ESS was also reduced more in the subgroup analysis of moderate OSA patients (MD: $-2.40 ; 95 \% \mathrm{CI}:-3.63$ to $-1.17 ; Z=3.83, P=0.0001$ ) and severe OSA patients (MD: $-4.64 ; 95 \%$ CI: -5.35 to $-3.92 ; Z=12.72, P<0.00001)$. Besides, acupuncture had a beneficial effect on $\mathrm{LSaO}_{2}(\mathrm{MD}$ : 5.29 ; 95\% CI: 2.61 to $7.97 ; Z=3.86, P=0.0001)$. The outcome of $\mathrm{AHI}$ and $\mathrm{LSaO}_{2}$ yielded consistent results after sensitivity analysis, but the direction of the outcome of ESS was reversed. And the quality of evidence was mainly low to very low. Conclusions. Acupuncture therapy is effective for OSA patients in reducing AHI and ESS and in improving the $\mathrm{LSaO}_{2}$ of various severities, especially in moderate and severe OSA patients. High-quality trials are urgently needed.

\section{Introduction}

Obstructive sleep apnea (OSA) is a common sleep disorder causing excessive daytime sleepiness, increasing risk for motor vehicle accidents, and impairing quality of life, and it is closely associated with cardiovascular events, diabetes, and other diseases [1-4]. Approximately 936 million adults worldwide have mild to severe OSA and 425 million have moderate to severe OSA; the prevalence exceeds $50 \%$ in some countries, and China is the most affected [5]. OSA is associated with high economic and social burdens-the cost for diagnosis and treatment in the United States was up to $\$ 12.4$ billion in 2015 [6]. Because of the high prevalence, haz- ard, and economic burden, the prevention and treatment of OSA is of great importance. Currently, therapies for OSA have been designed and proposed to reduce the frequency of sleep-disordered breathing events, including continuous positive airway pressure (CPAP) [7], surgical procedures $[8]$, weight loss and lifestyle interventions $[9,10]$, and pharmacotherapy [11]. One traditional and novel intervention is the application of acupuncture [12].

Previous meta-analysis of acupuncture for OSA including 6 randomized controlled trials (RCTs) involving 362 patients was done by Lv et al. [13] and was published in 2016. The review [13] showed that acupuncture was more effective in improving the apnea-hypopnea index (AHI) 
and further improved the apnea index and hypopnea index. However, the review can only demonstrate the overall efficacy of acupuncture on OSA. Subgroup analysis of OSA severity was not performed, and the efficacy of acupuncture may vary from severity. Furthermore, several relevant studies on the efficacy of acupuncture for treating OSA have been published since 2016. Thus, we undertook a systematic review and meta-analysis of RCTs to evaluate the efficacy and safety of acupuncture for OSA according to the severity of the disease.

\section{Methods}

Our review was performed strictly with the recommendations of the PRISMA (Preferred Reporting Items for Systematic Reviews and Meta-Analyses statement) [14] guidelines.

2.1. Eligibility Criteria. Only RCTs were included in this review, and they had to meet all of the following criteria: (1) were performed in OSA adult patients with different grades of severity in accordance with AHI (individuals with AHI values of $5 \sim 15,16 \sim 30$, or more than 30 times per hour were considered to have mild, moderate, or severe OSA, respectively) [2, 15]; (2) assessed the effects of acupuncture (electroacupuncture, manual acupuncture, etc.) compared with a control group (sham acupuncture or CPAP or pharmacotherapy or receiving no treatment); and (3) included at least one of the following outcomes: apnea-hypopnea index (AHI), Epworth Sleepiness Score (ESS), lowest oxygen saturation $\left(\mathrm{LSaO}_{2}\right)$, and adverse effect.

Studies were excluded if they met the following criteria: (1) OSA as a complication; (2) repeated publications (data were only extracted from one study that was recently updated); and (3) acupuncture treatment was performed less than 7 times.

2.2. Data Sources and Search Strategy. A comprehensive search was performed up to July 2019 with no language restriction, including 8 databases (PubMed, Cochrane Library, EMBASE, Web of Science, China National Knowledge Infrastructure (CNKI), Chongqing VIP (CQVIP), Wanfang Data, and Chinese Biomedical Literature Database $(\mathrm{CBM})$ ). Medical subject heading terms $(\mathrm{MeSH})$ and free terms were combined in the process of searching. Moreover, the reference lists of all related articles were examined for relevant citations to ensure the comprehensiveness of the search.

The following key terms and their variations were used in the conduct of a search, including ("Sleep Apnea, Obstructive" OR "sleep apnea" OR "OSA" OR "obstructive sleep apnea" OR "sleep hypopnea" OR "upper airway resistance sleep apnea syndrome" OR "obstructive sleep apneahypopnea syndrome" OR "OSAHS" OR "OSAS") AND ("Acupuncture Therapy" OR "acupuncture" OR "electroacupuncture" OR "manual acupuncture" OR "needling" OR "elongated needling" OR "scalp needle"). Search strategies are presented in Appendix 1.

2.3. Study Selection and Data Extraction. We identified the articles independently according to inclusion and exclusion criteria, first through the title and abstract and afterwards through the full text. And we extracted data from the eligible studies on a standardized data extraction form. Data including author information, publication year, participants, intervention, comparator, and outcomes were extracted. Missing or inconsistent data were managed by contacting authors; if this was not possible, the data would not be included in the meta-analysis. Two authors (Jia Xu and Yijun Zhan) performed the work independently. Disagreement was solved by discussion or a third author (Jian Pei).

2.4. Assessment of Risk of Bias. We assessed the risk of bias with the Cochrane Risk of Bias Assessment Tool [16]. Considering the characteristic of acupuncture, it is difficult to blind both the acupuncture operator and patients. Thus, we only focused on whether the assessment of the outcomes was blinded. Two authors (Jia Xu and Yijun Zhan) independently assessed the risk of bias and resolved disputes with the third author (Jian Pei).

2.5. Data Synthesis Statistical Analysis. The summary effect size was estimated by using risk ratios (RR) with $95 \%$ confidence intervals (CI) for dichotomous outcomes and mean difference (MD) with 95\% CI for continuous outcomes. If the same outcome was measured with different methods or scales, we calculated standardized mean difference (SMD). The results were combined in a meta-analysis using RevMan 5.3 software. We applied a fixed effects model to summarize the results when heterogeneity was not relevant $\left(I^{2}<30 \%\right)$. Otherwise, a random effects model was used, since primary RCTs included OSA patients with different severities (mild, moderate, and severe) and this could lead to heterogeneity. Therefore, we separated them into subgroups in all metaanalyses. Sensitivity analysis involving deleting each study separately was carried out in order to assess the quality and consistency of the results and explore the robustness of the findings regarding the study quality and sample size.

2.6. Evaluation of the Quality of the Evidence. We evaluated the quality of evidence using GRADE [17] (Grading of Recommendation, Assessment, Development, and Evaluation). We summarized the evidence for each of the outcomes in the table which was filled with the summary of the estimated-risk and $95 \%$ confidence intervals. The quality of evidence of each outcome was ranged from high, moderate to low, and very low.

\section{Results}

3.1. Literature Search and Study Selection. There were 873 articles in total. One was from a reference list, while the others were identified according to the search strategy. After reviewing the titles and abstracts, 272 duplicated and 518 irrelevant studies were excluded, and 83 potentially relevant studies remained for further assessment. Of these 83 studies, 74 were excluded, leaving 9 eligible randomized controlled trials $[12,18-25]$. The study selection process is shown in Figure 1 and the excluded articles are shown in Appendix 2. 


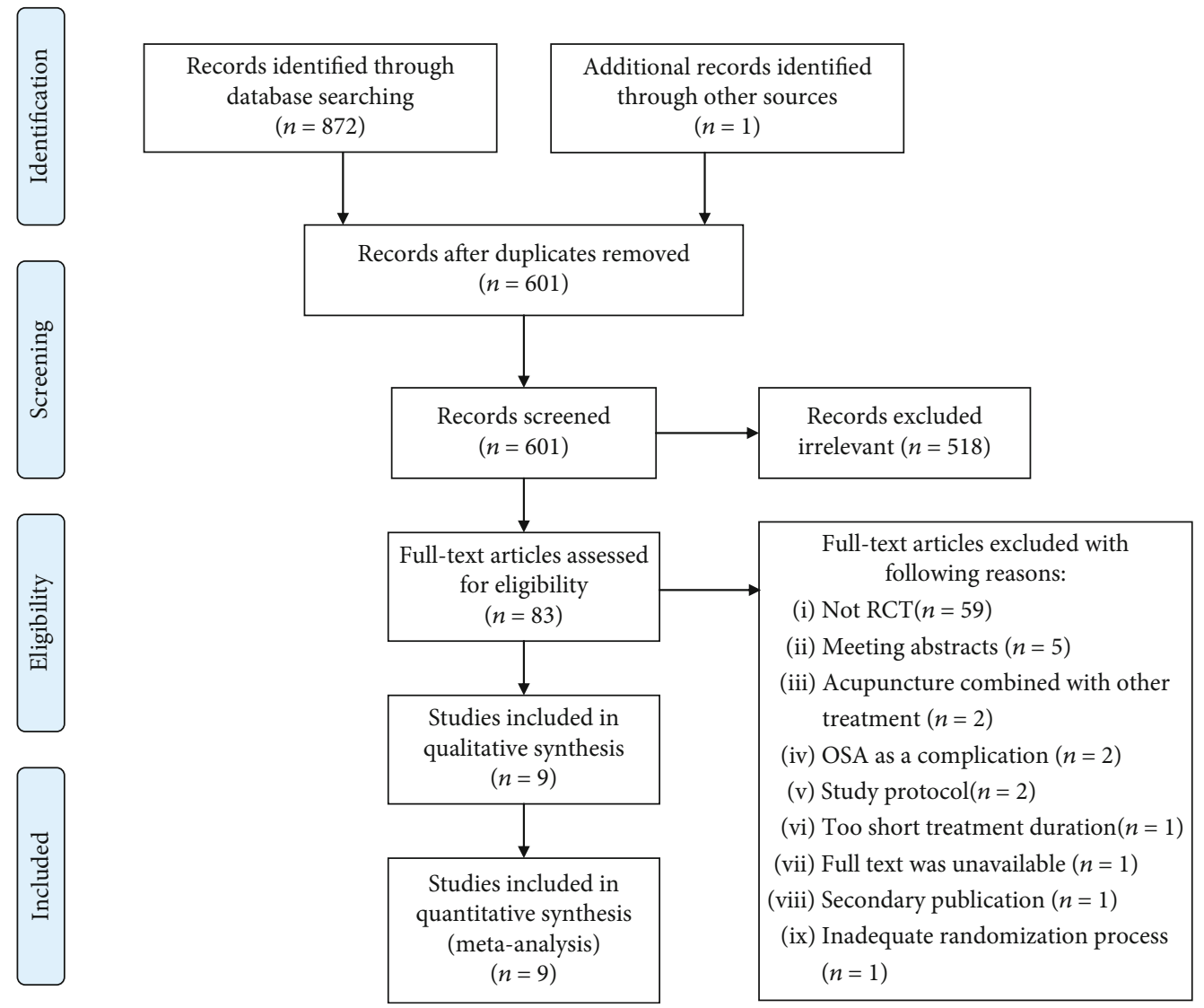

FIGURE 1: The study selection process.

3.2. Literature Characteristics. All included studies were RCTs published from 2007 to 2018. One study [12] was conducted in Brazil, and the other eight studies [18-25] were conducted in China. The nine studies included a total of 584 patients, with 304 in the acupuncture group and 280 in the control group. A detailed description of the characteristics of the included studies are shown in Table 1.

3.3. Risk of Bias Assessment. Included studies were assessed according to the Cochrane "Risk of Bias" Assessment Tool. Most of the trials are unclear and have a high risk of bias. All of the included studies mentioned randomization. In six studies $[12,18,21,23-25]$, participants were randomly assigned by a random number table. However, three studies $[19,20,22]$ failed to report the method of random sequence generation. Only one study [12] reported the methods of allocation concealment, and the blinding of participants and personnel was carried. Patients were unblind in the other eight studies because of the characteristic of intervention. The outcome assessors and statisticians were blinded in one study [12]. Two trials [12, 25] reported dropouts, and three studies [12, 22, 23] reported follow-up. Two studies $[22,24]$ failed to report outcomes in protocol, and the risk of bias were unclear. All studies did not mention other risks such as factory funding, so the risks of bias were unclear. The details of risk of bias assessment were presented in Figures 2 and 3.

\subsection{Effects of Interventions}

3.4.1. AHI. Nine studies $[12,18-25]$ reported AHI as an outcome. Significant heterogeneity was observed $\left(\chi^{2}=285.40\right.$, $\left.P<0.00001 ; I^{2}=94 \%\right)$. Therefore, a random-effect model was used. The analysis showed a significant treatment effect on AHI (MD: $-6.18 ; 95 \% \mathrm{CI}$ : -9.58 to $-2.78 ; Z=3.56$ $P=0.0004)$. Compared with mild OSA patients (MD: -1.78 ; 95\% CI: -2.99 to $-0.56 ; Z=2.86, P=0.004)$, AHI decreased more in subgroup analysis in moderate patients (MD: -9.44; 95\% CI: -12.44 to $-6.45 ; Z=6.18, P<0.00001)$ and severe patients (MD: $-10.09 ; 95 \% \mathrm{CI}:-12.47$ to $-7.71 ; Z=8.31, P<$ 0.00001 ) (Figure 4).

3.4.2. ESS. Four studies [19, 21, 22, 25] reported ESS as an outcome. Significant heterogeneity was observed $\left(\chi^{2}=28.4\right.$, $\left.P<0.00001 ; I^{2}=89 \%\right)$. Therefore, a random-effect model was used. The pooled results showed that ESS in the experimental group decreased more compared to the control group (MD: $-2.84 ; 95 \% \mathrm{CI}:-4.80$ to $-0.16 ; Z=2.09, P=0.04)$. In subgroup analysis, there was no significant decrease in ESS in mild patients (MD: 1.10 ; $95 \% \mathrm{CI}:-1.18$ to $3.38 ; Z=0.94$, $P=0.35)$, while ESS decreased significantly in moderate patients (MD: $-2.40 ; 95 \% \mathrm{CI}:-3.63$ to $-1.17 ; Z=3.83, P$ $=0.0001)$ and severe patients (MD: $-4.64 ; 95 \% \mathrm{CI}:-5.35$ to -3.92; $Z=12.72, P<0.00001$ ) (Figure 5). 


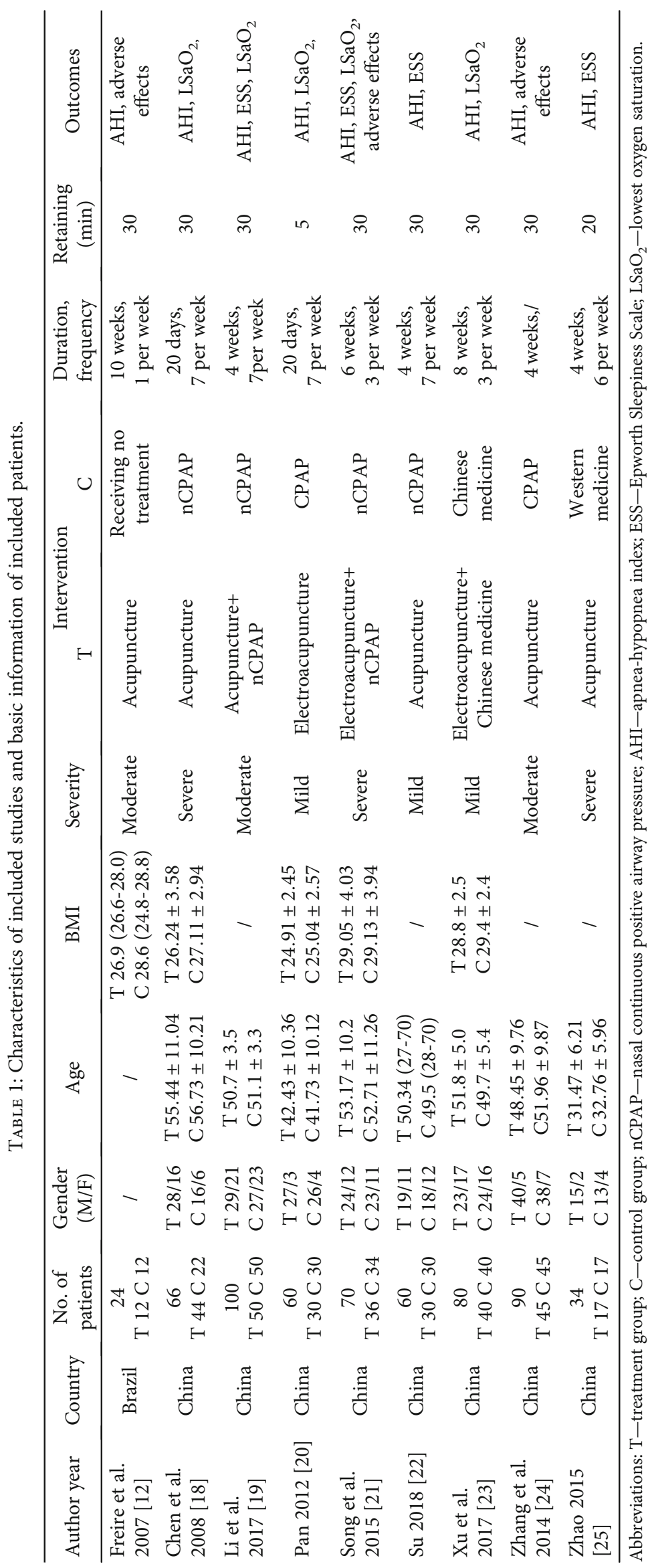




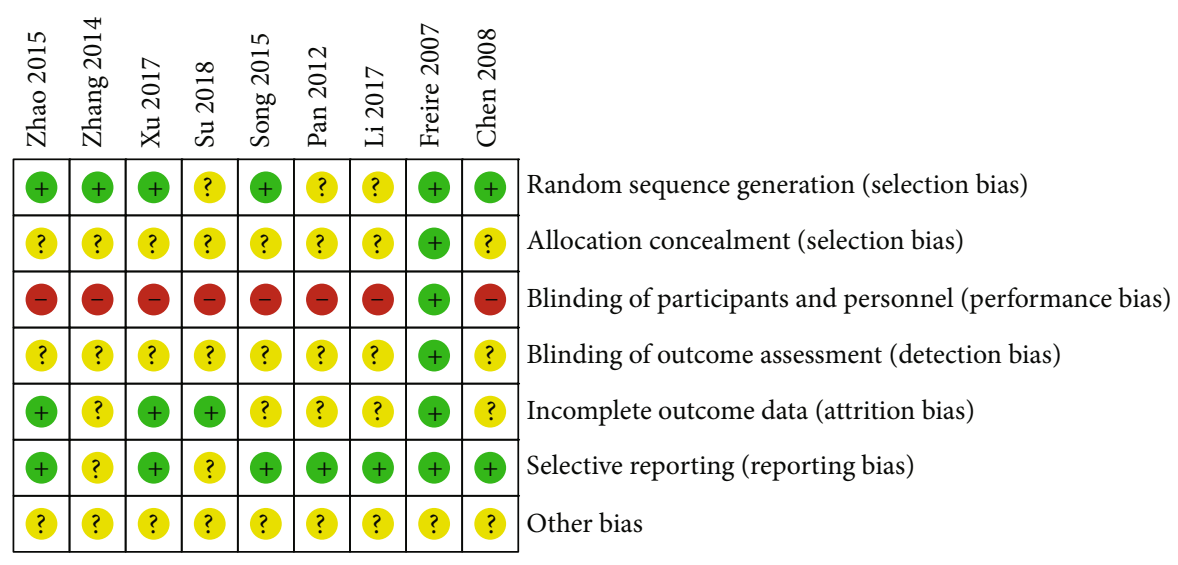

FIGURE 2: Risk of bias item for each included study.

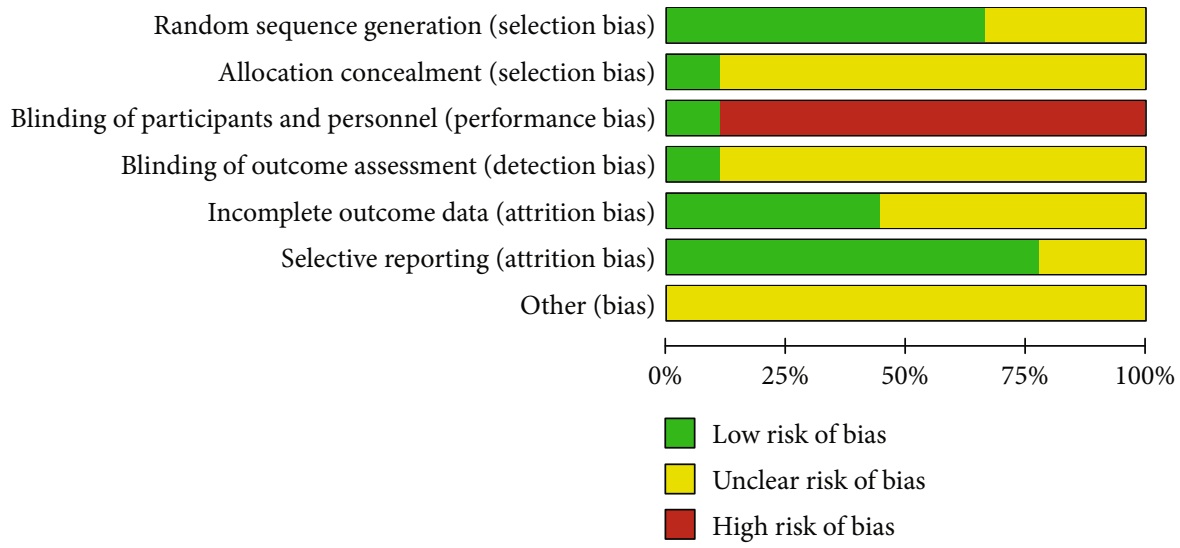

FIGURE 3: Risk of bias item presented as percentages across all included studies.

3.4.3. $\mathrm{LSaO}_{2}$. Five studies $[18-21,23]$ reported $\mathrm{LSaO}_{2}$ as an outcome. Significant heterogeneity was observed $\left(\chi^{2}=12.88\right.$, $\left.P=0.01 ; I^{2}=69 \%\right)$. Therefore, a random-effect model was used. The pooled results showed that $\mathrm{LSaO}_{2}$ in the experimental group improved more compared to the control group (MD: $5.29 ; 95 \% \mathrm{CI}: 2.61$ to $7.97 ; Z=3.86, P=$ $0.0001)$. In subgroup analysis, there was no significant decrease in $\mathrm{LSaO}_{2}$ in severe OSA patients (MD: 4.62; 95\% CI: -5.72 to $14.97 ; Z=0.88, P=0.38)$. $\mathrm{LSaO}_{2}$ decreased significantly in mild OSA patients (MD: 4.81 ; $95 \%$ CI: 2.49 to 7.14; $Z=4.06, P<0.0001)$ and moderate OSA patients (MD: $8.10 ; 95 \% \mathrm{CI}: 2.33$ to $13.87 ; Z=2.75, P=0.006$ ) (Figure 6).

3.5. Adverse Effect. There were some possible adverse effects of acupuncture, for instance, infection, hematoma, fainting during acupuncture, and bending of needle. In this review, three studies $[12,21,24]$ provided information about adverse effects, but no adverse effects were reported about acupuncture.

3.6. Publication Biases. We have conducted a comprehensive literature retrieval in the eight databases; conference papers, degree papers, and other documents were also within the scope of retrieval, with no language limitation, in order to minimize the possibility of publication bias. Nevertheless, we could not completely rule out the possibility of publication bias.

3.7. Sensitivity Analysis. The data were reanalyzed by deleting each study individually. Most of the outcomes yielded consistent results. However, after excluding the study conducted by Li et al. in 2017 or Song et al. in 2015 or Zhao in 2015 on ESS, the direction of the outcome was reversed (Table 2).

3.8. Quality of Evidence. The quality of evidence supporting the main outcome AHI was low. The evidence supporting the efficacy of acupuncture in ESS and $\mathrm{LSaO}_{2}$ was very low (Table 3).

\section{Discussion}

4.1. Main Findings. This is a further systematic review and meta-analysis to evaluate the efficacy and safety of acupuncture for OSA in various severities. We identified 9 RCTs of acupuncture for OSA, and these studies included 584 patients. With regard to the comparison, our meta-analysis of 9 RCTs showed that acupuncture significantly reduced the AHI of OSA, especially in moderate and severe OSA 


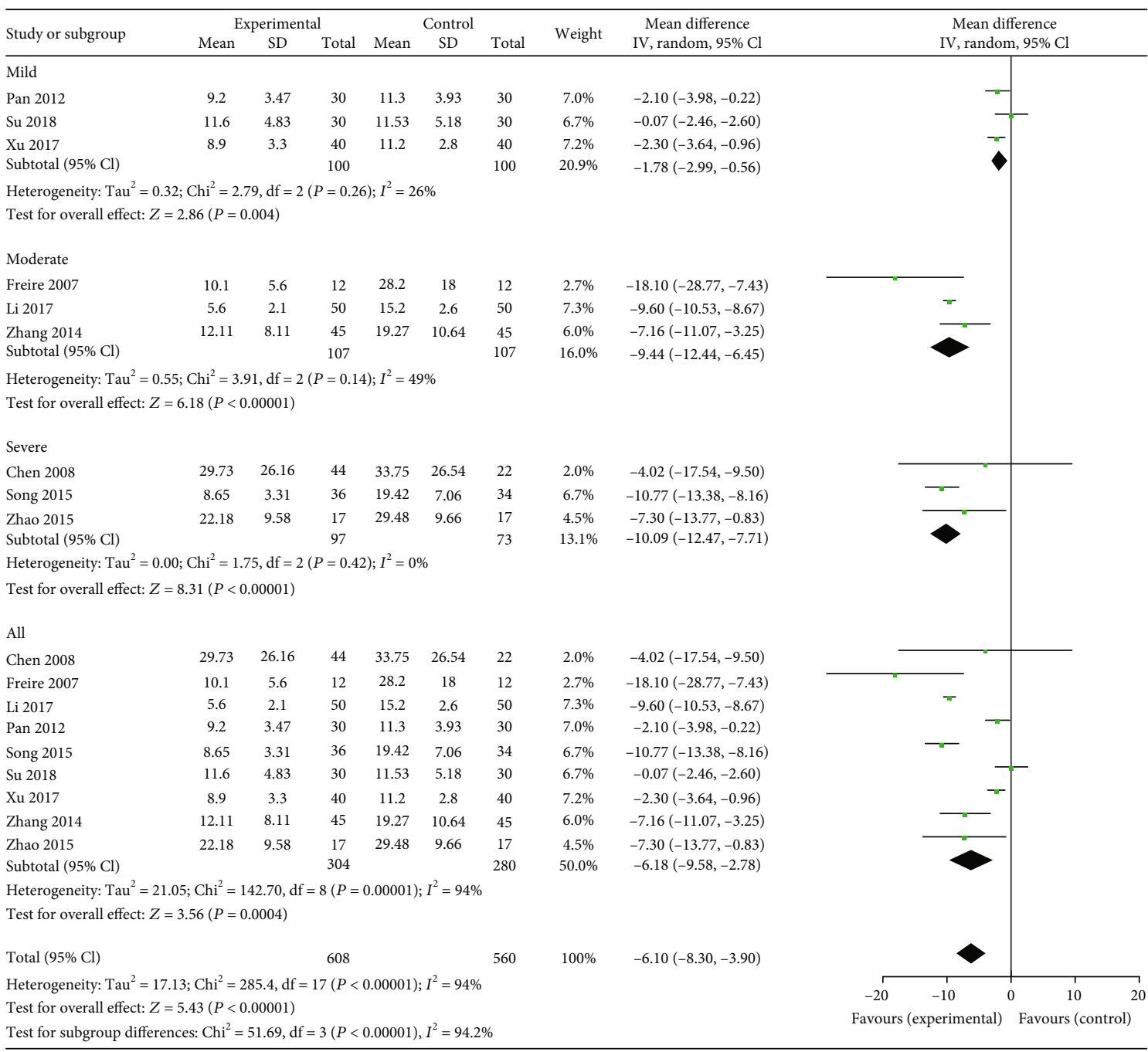

FIgURE 4: Experimental group versus control group, AHI.

\begin{tabular}{|c|c|c|c|c|c|c|c|c|c|c|c|c|}
\hline \multirow{3}{*}{$\begin{array}{l}\text { Study or subgroup } \\
\text { Mild }\end{array}$} & \multicolumn{3}{|c|}{ Experimental } & \multicolumn{3}{|c|}{ Control } & \multirow{2}{*}{ Weight } & \multirow{2}{*}{$\begin{array}{c}\text { Mean difference } \\
\text { IV, random, } 95 \% \mathrm{Cl}\end{array}$} & \multirow{2}{*}{\multicolumn{4}{|c|}{$\begin{array}{c}\text { Mean difference } \\
\text { IV, random, } 95 \% \mathrm{Cl}\end{array}$}} \\
\hline & Mean & SD & Total & Mean & SD & Total & & & & & & \\
\hline & & & & & & & & & & & & \\
\hline Su 2018 & \multirow[t]{2}{*}{12.3} & \multirow[t]{2}{*}{3.8} & 30 & \multirow[t]{2}{*}{11.2} & \multirow[t]{2}{*}{5.13} & 30 & $23.3 \%$ & $1.10(-1.18,38)$ & & & & \\
\hline Subtotal $(95 \% \mathrm{Cl})$ & & & 30 & & & 30 & $23.3 \%$ & $1.10(-1.18,3.38)$ & & & & \\
\hline \multicolumn{13}{|c|}{ Heterogeneity: Not applicable } \\
\hline \multicolumn{13}{|c|}{ Test for overall effect: $Z=0.94(P=0.35)$} \\
\hline \multicolumn{13}{|l|}{ Moderate } \\
\hline Li 2017 & \multirow[t]{2}{*}{5} & \multirow[t]{2}{*}{2.1} & 50 & \multirow[t]{2}{*}{7.4} & \multirow[t]{2}{*}{3.9} & 50 & $27.8 \%$ & $-2.40(-3.63,-1.17)$ & & & & \\
\hline Subtotal $(95 \% \mathrm{Cl})$ & & & 50 & & & 50 & $27.8 \%$ & $-2.40(-3.63,-1.17)$ & & & & \\
\hline \multicolumn{13}{|c|}{ Heterogeneity: Not applicable } \\
\hline \multicolumn{13}{|c|}{ Test for overall effect: $\mathrm{Z}=3.83(\mathrm{P}=0.0001)$} \\
\hline \multicolumn{13}{|l|}{ Severe } \\
\hline Song 2015 & 2.93 & 1.52 & 36 & 7.63 & 1.61 & 34 & $29.2 \%$ & $-4.70(-5.43,-3.97)$ & & - & & \\
\hline Zhao 2015 & \multirow[t]{2}{*}{10.51} & \multirow[t]{2}{*}{4.7} & 17 & \multirow[t]{2}{*}{14.02} & \multirow[t]{2}{*}{4.42} & 17 & $19.7 \%$ & $-3.51(-6.58,-0.44)$ & & & & \\
\hline Subtotal $(95 \% \mathrm{Cl})$ & & & 53 & & & 51 & $48.9 \%$ & $-4.64(-5.35,-3.92)$ & & & & \\
\hline \multicolumn{13}{|c|}{ Heterogeneity: $\mathrm{Tau}^{2}=0.00 ; \mathrm{Chi}^{2}=0.55, \mathrm{df}=1(P=0.46) ; I^{2}=0 \%$} \\
\hline \multicolumn{13}{|c|}{ Test for overall effect: $Z=12.72(P<0.00001)$} \\
\hline Total $(95 \% \mathrm{Cl})$ & & & 133 & & & 131 & $100.0 \%$ & $-2.48(-4.80,-0.16)$ & & & & \\
\hline \multicolumn{9}{|c|}{ Heterogeneity: $\mathrm{Tau}^{2}=4.65 ; \mathrm{Chi}^{2}=28.40, \mathrm{df}=3(P<0.00001) ; I^{2}=89 \%$} & -10 & -5 & 0 & $\sqrt{10}$ \\
\hline \multicolumn{9}{|c|}{ Test for overall effect: $Z=2.09(P=0.04)$} & \multicolumn{4}{|c|}{ Test for subgroup differences: $\mathrm{Chi}^{2}=27.85, \mathrm{df}=2(P<0.00001), I^{2}=92.8 \%$} \\
\hline
\end{tabular}

FIGURE 5: Experimental group versus control group, ESS. 


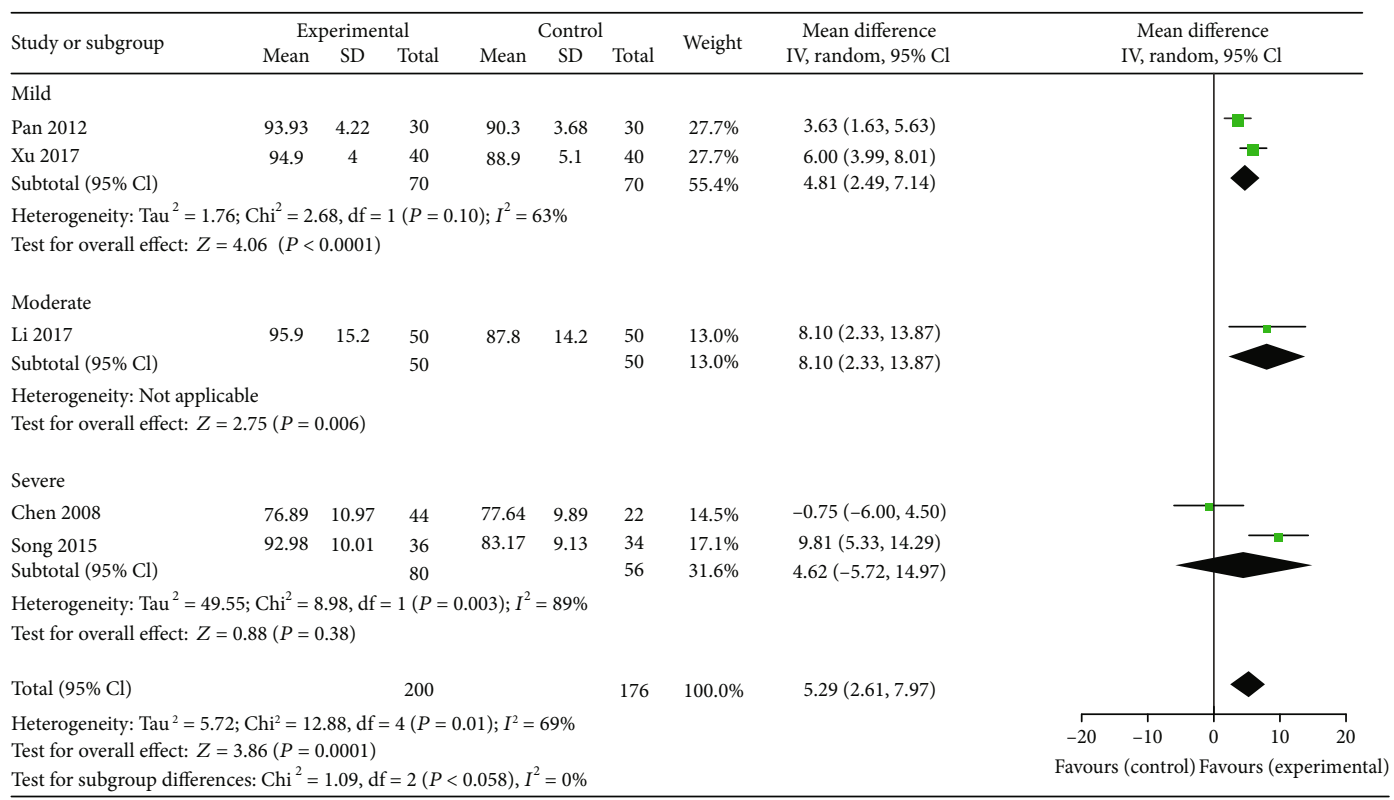

FIgURE 6: Experimental group versus control group, $\mathrm{LSaO}_{2}$.

TABLE 2: Results of sensitivity analysis.

\begin{tabular}{|c|c|c|c|}
\hline \multirow[t]{2}{*}{ Outcome } & \multirow{2}{*}{$\begin{array}{c}\text { Deletion } \\
\text { Chen et al. } 2008\end{array}$} & \multicolumn{2}{|c|}{ Result } \\
\hline & & $\chi^{2}=142.58, P<0.00001, I^{2}=95 \%$ & MD-6.28, 95\% CI: -9.77 to -2.79 \\
\hline \multirow{8}{*}{ AHI } & Freire et al. 2007 & $\chi^{2}=138.07, P<0.00001, I^{2}=95 \%$ & MD-5.42, $95 \%$ CI: -8.90 to -1.95 \\
\hline & Li et al. 2017 & $\chi^{2}=54.56, P<0.00001, I^{2}=87 \%$ & MD-5.39, $95 \%$ CI: -8.49 to -2.30 \\
\hline & Pan 2012 & $\chi^{2}=119.72, P<0.00001, I^{2}=94 \%$ & MD- $6.84,95 \%$ CI: -10.59 to -3.10 \\
\hline & Song et al. 2015 & $\chi^{2}=131.27, P<0.00001, I^{2}=95 \%$ & MD-5.50, $95 \%$ CI: -9.20 to -1.79 \\
\hline & Su. 2018 & $\chi^{2}=115.86, P<0.00001, I^{2}=94 \%$ & MD-7.10, 95\% CI: -10.62 to -3.58 \\
\hline & Xu et al. 2017 & $\chi^{2}=95.82, P<0.00001, I^{2}=93 \%$ & MD- $6.82,95 \%$ CI: -10.55 to -3.09 \\
\hline & Zhang et al. 2014 & $\chi^{2}=142.56, P<0.00001, I^{2}=95 \%$ & MD- $6.07,95 \%$ CI: -9.78 to -2.35 \\
\hline & Zhao 2015 & $\chi^{2}=142.63, P<0.00001, I^{2}=95 \%$ & MD-6.07, 95\% CI: -9.68 to -2.46 \\
\hline \multirow{4}{*}{ ESS } & Li et al. 2017 & $\chi^{2}=22.60, P<0.0001, I^{2}=91 \%$ & MD- $2.43,95 \%$ CI: -6.24 to 1.38 \\
\hline & Song et al. 2015 & $\chi^{2}=8.30, P=0.02, I^{2}=76 \%$ & MD- $1.55,95 \%$ CI: -4.05 to 0.94 \\
\hline & Su 2018 & $\chi^{2}=10.06, P=0.007, I^{2}=80 \%$ & MD-3.60, $95 \%$ CI: -5.40 to -1.80 \\
\hline & Zhao 2015 & $\chi^{2}=28.38, P<0.00001, I^{2}=93 \%$ & MD-2.19, 95\% CI: -4.97 to 0.58 \\
\hline \multirow{5}{*}{$\mathrm{LSaO}_{2}$} & Chen et al. 2008 & $\chi^{2}=7.90, P=0.05, I^{2}=62 \%$ & MD- $6.15,95 \%$ CI: 3.67 to 8.62 \\
\hline & Li et al. 2017 & $\chi^{2}=11.75, P=0.008, I^{2}=74 \%$ & MD- $4.86,95 \%$ CI: 1.89 to 7.83 \\
\hline & Pan 2012 & $\chi^{2}=9.63, P=0.02, I^{2}=69 \%$ & MD-5.89, $95 \%$ CI: 2.18 to 9.61 \\
\hline & Song et al. 2015 & $\chi^{2}=8.17, P=0.04, I^{2}=63 \%$ & MD- $4.39,95 \%$ CI: 1.80 to 6.99 \\
\hline & Xu et al. 2017 & $\chi^{2}=11.43, P=0.010, I^{2}=74 \%$ & MD-5.10, 95\% CI: 1.06 to 9.15 \\
\hline
\end{tabular}

patients. To our acknowledgement, AHI is used to classify the severity of the disease; people with an AHI value of $5 \sim 15,16 \sim 30$, or more than 30 times per hour are considered to have mild, moderate, or severe obstructive sleep apnea, respectively $[2,15]$. Similarly, AHI is also an important outcome of the efficacy of OSA, but there is no clinically signif- icant threshold of the AHI, and we could not determine the clinical curative effect of acupuncture in the treatment of OSA [1]; however, our meta-analysis results still gave us confidence, since acupuncture could effectively reduce AHI times per hour in OSA and reduce the frequency of sleepdisordered breathing events. Besides, ESS decreased 
TABLE 3: Quality of evidence evaluated by grading of recommendation assessment, development, and evaluation.

\begin{tabular}{|c|c|c|c|c|c|c|c|c|c|}
\hline \multirow{2}{*}{ Outcome } & \multirow{2}{*}{$\begin{array}{c}\text { Number of } \\
\text { studies }\end{array}$} & \multirow{2}{*}{$\begin{array}{c}\text { Sample } \\
\text { size }\end{array}$} & \multirow{2}{*}{$\begin{array}{c}\text { Confidence } \\
\text { intervals } \\
(95 \%)\end{array}$} & \multicolumn{5}{|c|}{ GRADE } & \multirow[t]{2}{*}{$\begin{array}{c}\text { Evidence } \\
\text { quality }\end{array}$} \\
\hline & & & & Limitation & Inconsistency & Indirections & Imprecision & $\begin{array}{l}\text { Publication } \\
\text { bias }\end{array}$ & \\
\hline AHI & 9 & 584 & $\begin{array}{c}\text { MD: }-6.18 ; \\
95 \% \text { CI: }-9.58 \\
\text { to }-2.78\end{array}$ & -1 & -1 & 0 & 0 & 0 & Low \\
\hline ESS & 4 & 264 & $\begin{array}{c}\text { MD: }-2.84 \\
95 \% \text { CI: }-4.80 \\
\text { to }-0.16\end{array}$ & -1 & -1 & 0 & -1 & 0 & Very low \\
\hline $\mathrm{LSaO}_{2}$ & 5 & 376 & $\begin{array}{l}\text { MD: } 5.29 ; \\
95 \% \text { CI: } 2.61 \\
\text { to } 7.97\end{array}$ & -1 & -1 & 0 & -1 & 0 & Very low \\
\hline
\end{tabular}

significantly in moderate and severe OSA patients in the acupuncture group, which exceeded the clinical significance threshold (2 points) [1]. Furthermore, the acupuncture group was superior to the control group in improving $\mathrm{LSaO}_{2}$. Meanwhile, nine RCTs did not report side effects due to acupuncture intervention. Most of the outcomes yielded consistent results after sensitivity analysis. However, the direction of the outcome of ESS was reversed after excluding the studies, which suggested that the results of ESS was unstable. The quality of evidence evaluated by GRADE in the included studies was mainly low to very low. High-quality trials are urgently needed.

4.2. Comparison to Previous Review. The previous review included 6 RCTs for analysis, including a total of 362 OSA patients, and revealed that both manual acupuncture (MA) and electroacupuncture (EA) were effective in improving AHI and mean $\mathrm{SaO}_{2}$, and MA could further improve the apnea index and the hypopnea index when compared with the control group; however, no definite conclusion could be drawn due to the limited evidence [13]. Our review did not include two RCTs $[26,27]$ that were included in the previous review, because one of them provided data on cerebrovascular disease patients [26] and the treatment duration of acupuncture for OSA did not meet the inclusion criteria in the other one [27]. In order to produce robust results, we performed rigorous inclusion criteria and included only RCTs that clearly stated the enrollment of patients specifically referred to OSA. Based on the previous review, we included another 5 RCTs $[19,20,22,23,25]$, which not only added OSA subjects, but also made our evidence more robust. In addition, our review was aimed at evaluating the effectiveness of acupuncture on three different degrees (mild, moderate, and severe) of OSA, which would be closer to clinical practice, making it easier for physicians to make decisions.

4.3. Advantages of Acupuncture Treatment. We recommend acupuncture as an adjuvant therapy for OSA because it has some of the following advantages. Acupuncture has an acute effect in reducing AHI as well as the number of nocturnal respiratory events of OSA patients [27], which can play a therapeutic effect on OSA patients more rapidly. At present, the most effective therapy to reduce OSA is positive airway pressure (PAP), and CPAP provides a constant level of positive pressure across inspiration and expiration [28]. However, it requires tremendous effort on the patient's part to position the mask properly and maintain the machine and supplies. And direct side effects have been reported with the use of PAP, including headache, chest discomfort, and sense of suffocation or difficulty exhaling. These side effects can result in sleep disruption and poor sleep quality, thereby reducing patient adherence to CPAP [29]. Thus, a complementary therapy such as acupuncture may be warranted when treatment intolerance due to side effects occurs. Besides, compared with acupuncture, CPAP has the greater cost-effectiveness, while acupuncture has advantage in cost. Furthermore, all studies included mentioned the arrival of qi when acupuncture was performed and the retention of the needle. The most commonly used acupoints were Zhaohai (KI6), Sanyinjiao (SP6), Sishencong (EX-HN1), Shenmen (HT7), Zusanli (ST36), and lianquan (CV23). Point selection and arrival of qi were essential in the acupuncture treatment for OSA. Acupuncture plays an effective role only if the operating procedure was observed.

4.4. Limitations and Weaknesses. There were some limitations in this review. Firstly, acupuncture treatment for OSA has some bias and heterogeneity. The reasons may be the various interventions of acupuncture. Secondly, in this review, OSA patients were categorized as mild, moderate, and severe according to AHI before treatment. AHI is an important measure which is used to diagnose and categorize disease severity of OSA patients. However, there are inherent limitations with using the AHI calculated from one night of sleep to categorize disease severity, because the AHI is influenced by many factors and may vary over time and even across consecutive nights [2]. Thirdly, the methodological quality of the included RCTs was generally low. For example, most of the included studies had a high risk of performance bias.

\section{Conclusions}

Acupuncture may be an effective and safe treatment in OSA patients. Besides, acupuncture may also reduce AHI and ESS and improve $\mathrm{LSaO}_{2}$ of OSA patients. This result is more significant in moderate and severe OSA patients. These 
evidences may be useful to clinicians, patients, and health policy makers with regard to the application of acupuncture in OSA. However, further high-quality RCTs are needed to confirm the efficacy and safety of acupuncture for OSA patients.

\section{Conflicts of Interest}

The authors declare that there are no conflicts of interest.

\section{Authors' Contributions}

Liaoyao Wang searched the literature, conducted the statistical analysis, and drafted the manuscript. Jia Xu and Yijun Zhan screened the studies, extracted the data, and evaluated the risk of bias. Jia Xu revised the manuscript. Jian Pei conceived this study.

\section{Acknowledgments}

This study was supported by the Key Scientific Research Program of the Shanghai Municipal Science and Technology Committee in China (16401970300 and 18401970500), the Financing Scheme of Arising Interdisciplinary Subjects of TCM in Shanghai (Shxxjcxk201709), and the TCM Genre Program of Shanghai Health Bureau (ZY (2018-2020)CCCX-1006).

\section{Supplementary Materials}

Appendix 1 shows the details of the literature search strategy. Appendix 2 shows the basic information of the excluded articles. (Supplementary Materials)

\section{References}

[1] V. K. Kapur, D. H. Auckley, S. Chowdhuri et al., "Clinical practice guideline for diagnostic testing for adult obstructive sleep apnea: an American Academy of Sleep Medicine clinical practice guideline," Journal of Clinical Sleep Medicine, vol. 13, no. 3, pp. 479-504, 2017.

[2] S. C. Veasey and I. M. Rosen, "Obstructive sleep apnea in adults," The New England Journal of Medicine, vol. 380, no. 15, pp. 1442-1449, 2019.

[3] T. Kawada, "Obstructive sleep apnea and cognitive decline in older adults," American Journal of Respiratory and Critical Care Medicine, vol. 199, no. 9, pp. 1168-1169, 2019.

[4] A. Subramanian, N. J. Adderley, A. Tracy et al., "Risk of incident obstructive sleep apnea among patients with type 2 diabetes," Diabetes Care, vol. 42, no. 5, pp. 954-963, 2019.

[5] A. V. Benjafield, N. T. Ayas, P. R. Eastwood et al., "Estimation of the global prevalence and burden of obstructive sleep apnoea: a literature-based analysis," The Lancet Respiratory Medicine, vol. 7, no. 8, pp. 687-698, 2019.

[6] N. F. Watson, "Health care savings: the economic value of diagnostic and therapeutic care for obstructive sleep apnea," Journal of Clinical Sleep Medicine, vol. 12, no. 8, pp. 10751077, 2016.

[7] F. da Silva Paulitsch and L. Zhang, "Continuous positive airway pressure for adults with obstructive sleep apnea and car- diovascular disease: a meta-analysis of randomized trials," Sleep Medicine, vol. 54, pp. 28-34, 2019.

[8] S. M. Caples, J. A. Rowley, J. R. Prinsell et al., "Surgical modifications of the upper airway for obstructive sleep apnea in adults: a systematic review and meta-analysis," Sleep, vol. 33, no. 10, pp. 1396-1407, 2010.

[9] A. Carneiro-Barrera, A. Díaz-Román, A. Guillén-Riquelme, and G. Buela-Casal, "Weight loss and lifestyle interventions for obstructive sleep apnoea in adults: systematic review and meta-analysis," Obesity Reviews, vol. 20, no. 5, pp. 750-762, 2019.

[10] D. W. Hudgel, S. R. Patel, A. M. Ahasic et al., "The role of weight management in the treatment of adult obstructive sleep apnea. An official American Thoracic Society clinical practice guideline," American Journal of Respiratory and Critical Care Medicine, vol. 198, no. 6, pp. e70-e87, 2018.

[11] T. Gaisl, S. R. Haile, S. Thiel, M. Osswald, and M. Kohler, "Efficacy of pharmacotherapy for OSA in adults: a systematic review and network meta-analysis," Sleep Medicine Reviews, vol. 46, pp. 74-86, 2019.

[12] A. O. Freire, G. C. M. Sugai, F. S. Chrispin et al., "Treatment of moderate obstructive sleep apnea syndrome with acupuncture: a randomised, placebo-controlled pilot trial," Sleep Medicine, vol. 8, no. 1, pp. 43-50, 2007.

[13] Z. T. Lv, W. X. Jiang, J. M. Huang, J. M. Zhang, and A. M. Chen, "The clinical effect of acupuncture in the treatment of obstructive sleep apnea: a systematic review and metaanalysis of randomized controlled trials," Evidence-Based Complementary and Alternative Medicine, vol. 2016, Article ID 8792167, 10 pages, 2016.

[14] A. Liberati, D. G. Altman, J. Tetzlaff et al., "The PRISMA statement for reporting systematic reviews and meta-analyses of studies that evaluate healthcare interventions: explanation and elaboration," British Medical Journal, vol. 339, article b2700, 2009.

[15] W. R. Ruehland, P. D. Rochford, F. J. O’Donoghue, R. J. Pierce, P. Singh, and A. T. Thornton, "The new AASM criteria for scoring hypopneas: impact on the apnea hypopnea index," Sleep, vol. 32, no. 2, pp. 150-157, 2009.

[16] J. P. T. Higgins, D. G. Altman, P. C. Gotzsche et al., "The Cochrane Collaboration's tool for assessing risk of bias in randomised trials," British Medical Journal, vol. 343, article d5928, 2011.

[17] G. H. Guyatt, A. D. Oxman, G. E. Vist et al., "GRADE: an emerging consensus on rating quality of evidence and strength of recommendations," BMJ, vol. 336, no. 7650, pp. 924-926, 2008.

[18] B. Chen, X. S. Zhang, H. Huang, Y. Jia, and X. M. Xie, “A study on differences of curative effects of acupuncture and nCPAP for treatment of OSAHS," Zhongguo Zhen Jiu, vol. 28, no. 2, pp. 79-83, 2008.

[19] X. X. Li, J. Q. Zhang, and W. G. Ding, "Clinical observation of obstructive sleep apnea hypopnea syndrome treated by consciousness-restoring and orifices-opening acupuncture," Henan Traditional Chinese Medicine, vol. 37, no. 5, pp. 879882, 2017.

[20] H. H. Pan, Acupuncture in the Treatment of Obstructive Sleep Apnea Syndrome Clinical Observation, Heilongjiang University of Traditional Chinses Medicine, 2012.

[21] Y. J. Song, W. J. Yu, T. Xu, and X. Gu, "Curative observation of the electro-acupuncture and nasal continuous positive airway 
pressure on patients with obstructive sleep apnea hypoventilations," Journal of Emergency in Traditional Chinese Medicine, vol. 24, no. 8, pp. 1352-1356, 2015.

[22] S. Su, "The curative effect observation of slow twisting needle method in treating obstructive sleep apnea hypopnea syndrome," Henan Traditional Chinese Medicine, vol. 38, no. 2, pp. 289-291, 2018.

[23] T. Xu, S. Wang, Y. J. Song, and W. J. Yu, "Efficacy of electroacupuncture for patients with mild obstructive sleep apnea hypopnea syndrome," Zhejiang Medical Journal, vol. 39, no. 16, pp. 1353-1358, 2017.

[24] L. X. Zhang, L. Shi, H. Y. Zhou et al., "Clinical observation on the effect of acupuncture in the treatment of 90 cases with obstructive sleep apnea," China Health Industry, vol. 11, no. 20, pp. 192-193, 2014.

[25] D. Zhao, The Clinical Observation and Mechanism Analysis of the Li Qi Qu Tan Acupuncture Therapy for the Qi-Stagnancy with Phlegm-Blocking Type OSAHS, Shandong University of Traditional Chinese Medicine, 2015.

[26] P. L. Zhang, "Treating 30 patients with obstructive sleep apnea hypopnea syndrome by laryngeal three acupoints," Western Journal of Traditional Chinese Medicine, vol. 27, no. 10, pp. 129-130, 2014.

[27] A. O. Freire, G. C. Sugai, S. M. Togeiro, L. E. Mello, and S. Tufik, "Immediate effect of acupuncture on the sleep pattern of patients with obstructive sleep apnoea," Acupuncture in Medicine, vol. 28, no. 3, pp. 115-119, 2010.

[28] S. P. Patil, I. A. Ayappa, S. M. Caples, R. J. Kimoff, S. R. Patel, and C. G. Harrod, "Treatment of adult obstructive sleep apnea with positive airway pressure: an American Academy of Sleep Medicine clinical practice guideline," Journal of Clinical Sleep Medicine, vol. 15, no. 2, pp. 335-343, 2019.

[29] S. P. Patil, I. A. Ayappa, S. M. Caples, R. J. Kimoff, S. R. Patel, and C. G. Harrod, "Treatment of adult obstructive sleep apnea with positive airway pressure: an American Academy of Sleep Medicine systematic review, meta-analysis, and GRADE assessment," Journal of Clinical Sleep Medicine, vol. 15, no. 2, pp. 301-334, 2019. 